\title{
Editorial: Special Issue on the Fifth Computational Visual Media Conference (CVM 2017)
}

\author{
Niloy J. Mitra ${ }^{1}$ Yizhou $\mathrm{Yu}^{2}$ Ming-Ming Cheng ${ }^{3}$ \\ ${ }^{1}$ University College London $\quad{ }^{2}$ The University of Hong Kong ${ }^{3}$ Nankai University
}

This special issue of Graphical Models includes a selection of oral papers presented at the recent Computational Visual Media conference held at Nankai University, Tianjin, China, April 12-14, 2017. The Computational Visual Media (CVM) conference series is intended to provide a major international forum for exchanging novel research ideas and significant practical results either underpinning or related to visual media. The CVM 2017 conference received a great deal of attention as evidenced by the record number of 168 complete submissions. Among these submissions, 27 have been accepted as oral papers and 4 oral papers have been recommended to be published in this special issue.

Gao et al. [1] extend previous as-rigid-as-possible surface deformation methods by making rigidity controllable. The main contribution is a novel rigidity controllable mesh deformation method, which can flexibly adjust shape rigidity by expanding the local neighborhood around a vertex beyond the first ring. This is supported by the observation that larger neighborhoods give rise to more rigid deformation. Different levels of rigidity for different parts of an object are useful for interactive deformation. The size of local neighborhoods can be either learned from existing deformation data or specified by the user.

Han et al. [2] present a fast propagation scheme for computing approximate single-source all-destinations geodesic paths over large-scale meshes. The proposed scheme optimizes an initial path by attempting to eliminate geodesic curvature everywhere on the path, avoiding the expensive back-tracing step. The resulting floating path is projected to the mesh surface to obtain the refined one for further propagation. This paper successfully integrates geodesic optimization with the continuous Dijkstra strategy, therefore, improves both the speed and memory usage of approximate geodesic path algorithms.

Yang et al. [3] propose a framework for tree modeling through the exploration of a high-level parameter space of tree models. The parameters of this space were learned from crowdsourced data with the goal of obtaining a set of semantically meaningful properties typically ascribed to trees. Exploration is carried out using an online learning procedure that iteratively updates a model of the user intent. In each round, a set of candidate trees are shown to the user and a chosen subset is used to update the model. To handle the exploration-exploitation trade-off, the authors use confidence bounds on the estimated user intent.

Mizrahi et al. [4] propose an approach for computing the Minkowski sum of two free-form surfaces, in the form of triangular meshes. Compared with existing techniques, the proposed method is able to handle more general free-form surfaces.

This special issue would not have been possible without the efforts of 80 distinguished program committee members and 149 reviewers of the CVM 2017 conference [5]. Most importantly, we thank all the authors for their contributions.

\section{References}

[1] L. Gao, S.-Y. Chen, and Y.-K. Lai, "Rigidity controllable as-rigid-as-possible shape deformation," Graphical Models, vol. 1, no. 1, pp. 1-1, 2017.

[2] X. Han, H. Yu, Y. Yu, and J. Zhang, "A fast propagation scheme for approximate geodesic paths," Graphical Models, vol. 1, no. 1, pp. 1-1, 2017.

[3] Y. Yang, R. Wang, H. Zhang, and H. Bao, "Exploretree:interactive tree modeling in semantic trait space with online intent learning," Graphical Models, vol. 1, no. 1, pp. 1-1, 2017. 
[4] J. Mizrahi, S. Kim, I. Hanniel, M.-S. Kim, and G. Elber, "Minkowski sum computation of b-spline surfaces," Graphical Models, vol. 1, no. 1, pp. 1-1, 2017.

[5] "Computational Visual Media Conference," http://iccvm.org/2017/, 2017. 